\title{
Jean-Marie Pillon, Pôle emploi. Gérer le chômage de
} masse

Rennes, Presses universitaires de Rennes, 2017

\section{Pascal Ughetto}

\section{OpenEdition}

\section{Journals}

Édition électronique

URL : http://journals.openedition.org/travailemploi/7730

DOI : $10.4000 /$ travailemploi. 7730

ISSN : 1775-416X

Éditeur

DARES - Ministère du Travail

Édition imprimée

Date de publication : 1 juillet 2017

Pagination : 101-105

ISSN : 0224-4365

\section{Référence électronique}

Pascal Ughetto, « Jean-Marie Pillon, Pôle emploi. Gérer le chômage de masse », Travail et Emploi [En ligne], 151 | juillet-septembre 2017, mis en ligne le 11 juillet 2019, consulté le 23 septembre 2020. URL : http://journals.openedition.org/travailemploi/7730; DOI : https://doi.org/10.4000/travailemploi. 7730 


\title{
Notes DE LECTURE
}

\section{Pôle emploi. Gérer le chômage de masse}

\author{
Jean-Marie Pillon
}

Rennes, Presses universitaires de Rennes, 2017, 272 p.

\section{Lu par Pascal Ughetto*}

Issu de sa thèse de doctorat, l'ouvrage que Jean-Marie Pillon consacre à Pôle emploi s'intéresse à la mesure de son efficacité - entendant « par-là la capacité de cet établissement à réaliser ses missions » (p. 11) - et à la façon dont il s'outille pour ce faire. En se fondant sur un grand volume d'observations et d'entretiens ${ }^{1}$, il rend compte du travail des contrôleurs de gestion en relation avec les personnels des agences et les managers. Après une introduction permettant au lecteur de saisir que la mesure de l'efficacité dans ses différentes dimensions a une histoire aussi longue que celle de l'organisme (de l'ANPE à Pôle emploi), l'ouvrage s'agence en trois parties de deux chapitres chacune.

Parcourant chronologiquement l'histoire de l'institution, le premier chapitre dément le présupposé selon lequel le sujet ici traité n'aurait surgi que récemment, sous l'effet des pressions exercées sur les opérateurs publics pour importer le modèle de l'entreprise. Bien qu'ayant eu des effets limités sur le travail, le comptage des offres collectées et la mesure de l'efficacité du rapprochement entre ces offres et les demandeurs d'emploi ont été mis en place dès la création de l'ANPE, en 1967, initialement pour répondre aux besoins exprimés par les commissions des quatre premiers Plans (1947-1966) et de la Rationalisation des choix budgétaires. L'entrée dans la période du chômage de masse, posant de nouveaux défis à l'organisme et à ses conseillers, engendre une nouvelle étape de production d'indicateurs, favorisée par les progrès de l'informatique. Entre 1980 et 1985, la direction des études et des statistiques de l'ANPE est elle-même à la manœuvre pour produire des mesures de la productivité à opposer aux reproches d'inefficacité qui lui sont faits. Elle concède ainsi aux autorités de tutelle qu'elle peut gagner en efficacité pour mieux argumenter en faveur d'une augmentation de ses moyens. Dès la fin des années 1980, c'est encore plus le cas avec les contrats de performance conclus avec l'État, lorsque l'ANPE devance le programme « Renouveau du service public » du gouvernement Rocard

\footnotetext{
* Université Paris-Est, Laboratoire techniques, territoires et sociétés (LATTS).

1. Responsables de l'évaluation au sein de l'organisme à différentes périodes de son histoire depuis la création de l'ANPE, contrôleurs de gestion à différents niveaux, développeurs de logiciels de gestion, membres de la hiérarchie, notamment dans les agences, conseillers, etc.
} 
en produisant un argumentaire fondé sur des données de performance à des fins de dialogue contractuel autour des enveloppes budgétaires. La généralisation progressive du vocabulaire et de la « grammaire » du contrôle de gestion accompagne l'affirmation du pouvoir d'une équipe de spécialistes au sein de l'établissement, qui se conclut par la création d'une direction à part entière. En 1992, remplaçant l'indicateur du nombre de placements en vigueur depuis 1971, les notions de «mise en relation » (MER) et de «mise en relation positive » (MER +, celle qui voit l'information donnée au demandeur d'emploi déboucher effectivement sur une embauche), entrent désormais dans le paysage quotidien des conseillers et de leur hiérarchie, afin de leur permettre de mesurer les effets des moyens accordés.

Le deuxième chapitre (1990-2008) relate la fusion avec l'Unedic, prévue pour favoriser le contrôle et l'accompagnement simultanés des chômeurs, ainsi qu'une activation des dépenses pour l'emploi - c'est-à-dire le fait de privilégier, dans ces dépenses, celles qui ne représenteraient pas qu'une indemnisation «passive » mais aideraient et inciteraient les individus à trouver un nouvel emploi. Décidée en 2008, cette fusion est engagée alors que la crise économique submerge le nouvel établissement de demandeurs d'emploi, faisant sombrer corps et biens le projet de forger un « métier unique » de conseiller chargé de l'accompagnement et de la liquidation des droits. Conjuguer dans une même activité de travail les tâches accomplies jusqu'alors dans des institutions différentes suppose d'énormes investissements en formation et du temps long, qui n'ont pas été réunis. Par un tour de passe-passe de communication, le gouvernement va se contenter de l'objectif d'un référent unique, simple rapprochement des métiers de l'accompagnement et de l'indemnisation : le référent unique est l'agent qui, maîtrisant l'ensemble des services proposés par l'institution, est le contact donnant au demandeur d'emploi ou à l'entreprise le premier niveau de réponse sur des sujets d'indemnisation ou de placement, tout en demeurant spécialiste de l'un ou l'autre des deux métiers. Les missions de recevoir et de conseiller se voient accorder la priorité par les indicateurs de suivi de la fusion, comme si le travail accompli par les personnels de l'Unedic n'était qu'une intendance technique, jusqu'à ce que la colère des demandeurs d'emploi mal indemnisés conduise à corriger le tir. « Les directeurs d'agence notifient à leur hiérarchie qu'ils n' arrivent plus à liquider assez vite les dossiers d'indemnisation » (p. 86) et un plan de recrutement de chargés d'accueil, en contrat temporaire, finit par être décidé pour soulager les agents permanents.

L'ouvrage se concentre ensuite sur les contrôleurs de gestion, au pouvoir ambigu. Le troisième chapitre expose ce qu'ils sont censés faire. Sans pouvoir hiérarchique direct, ils cherchent moins à imposer leur logique à la ligne managériale qu'à élaborer et négocier avec elle des objectifs, et à lui fournir des arguments chiffrés pour se défendre ou attaquer : mettre en valeur un chiffre favorable, préparer une explication pour un autre qui l'est moins. C'est dans le contrôle budgétaire que réside leur pouvoir. Artisans des mesures de la productivité, ils ont l'art de rapporter tout ce qui se fait dans les services à cet étalon qu'est le « temps agent ». Cependant, les jeux d'acteurs auxquels les instruments qu'ils mobilisent donnent lieu n'ont rien d'unilatéral : les 
contrôleurs de gestion doivent notamment faire un travail de conviction auprès des managers. On appréciera ainsi particulièrement dans ce chapitre la section consacrée aux outils de gestion Opera (destiné à prévoir la demande sociale, c'est-à-dire le volume anticipé d'entretiens d'inscription, de demandeurs d'emploi indemnisés ou non, ainsi que d'établissements déclarant une embauche, pour répartir équitablement les ressources entre régions) et Oscar (permettant d'apprécier si les effectifs d'une division territoriale de l'établissement sont suffisants pour atteindre les objectifs du contrat de performance).

Dans le prolongement de ce que la sociologie des organisations a tant décrit, le quatrième chapitre révèle comment cadres et conseillers évalués jouent avec le système d'information pour réaliser le travail selon leurs propres exigences tout en étant en conformité avec les objectifs mesurés. Les tableaux de bord sont peuplés de chiffres supposés être de fidèles reflets de l'activité, mais, de plus près, on s'aperçoit que les contrôleurs de gestion, les conseillers et les managers consacrent beaucoup d'énergie à produire des chiffres rendant justice à leur activité. Entrer l'information de manière stratégique ou par exemple, enregistrer des entretiens fictifs : ces actes confirment, comme on pouvait l'anticiper, que tous cherchent un compromis entre le travail réel et sa représentation chiffrée, y compris avec la collaboration des contrôleurs de gestion, d'abord soucieux d'enrôler les opérationnels dans l'utilisation du système d'information et de les y faire coopérer.

Les objectifs à atteindre affectent-ils le traitement des chômeurs ? C'est à cette question que s'intéressent les deux chapitres suivants qui répondent, en quelque sorte, « oui et non ». Le travail sur les dossiers des agents de Pôle emploi, décrit dans le cinquième chapitre, comporte une part importante de codage des caractéristiques des demandeurs d'emploi. Ce codage n'est pas neutre : il n'est ni technique ni mécanique, il dépend de ce que ces agents savent du codage des postes vacants, dans un contexte de sélectivité du marché du travail et avec l'impératif d'apporter la preuve que l'agence reçoit, oriente et place. «Il est possible de masquer le fait qu'une requête a été traitée de façon précipitée, en inscrivant les codes adaptés dans le dossier du chômeur. Il est possible, au contraire, de traiter la demande de façon adéquate, sans pour autant en faire mention dans le dossier, afin d'économiser le temps de saisie » (p. 145).

Le sixième et dernier chapitre montre en outre que le codage est effectué dans un univers où ont émergé des plateformes de traitement spécifique de populations ciblées, à distance du guichet et du flux de demandeurs d'emploi : plateformes téléphoniques dispensées de l'inscription des chômeurs et de leur suivi mensuel et donc entièrement concentrées sur l'intermédiation, ou spécialisées sur la méthode de recrutement par simulation. Sans échapper à la pression du chiffre - à l'image du taux de décroché (proportions d'appels téléphoniques débouchant sur un contact avec un conseiller après quelques sonneries), qui vise à conforter la qualité du service rendu aux employeurs -, ces plateformes agissent en prestataires des agences, mais sont aussi en concurrence avec elles pour capter les offres. "L'orientation d'une offre vers les plateformes constitue une perte nette pour l'agence locale de Pôle emploi. Dans la mesure où 
l'évaluation de l'efficacité est faite isolément, par site de production, la mise en place d'un recrutement selon la méthode de recrutement par simulation déplace le lieu d'enregistrement de l'efficacité de l'agence locale vers la plateforme » (p. 228).

Pas de découvertes révolutionnaires dans cet ouvrage, mais la documentation précieuse, avec précision et nuance, de phénomènes plus souvent condamnés que décortiqués avec rigueur. L'ouvrage de J.-M. Pillon est une contribution remarquable dans un ensemble extrêmement fourni de travaux qu'il vient compléter : les travaux sur la quantification d'Alain Desrosières, que l'auteur mobilise beaucoup ; L'Esprit gestionnaire d'Albert Ogien, dont il s'inspire tout en s'en démarquant par son soin à décrire les fonctionnements organisationnels et l'activité de travail, là où $\mathrm{A}$. Ogien ou des ouvrages plus récents, comme La Bureaucratisation du monde à l'ère néolibérale de Béatrice Hibou, s'attachent surtout aux effets sur l'action publique du recours croissant à la mesure et s'en inquiètent; la littérature prolifique sur le New Public Management ou encore la sociologie de la gestion. Il se range à côté des contributions qui documentent de près ces phénomènes, comme l'ouvrage de Nicolas Belorgey, L'Hôpital sous pression, ou Réinventer l'État de Philippe Bezes. Le grand mérite de l'ouvrage de J.-M. Pillon est de ne pas se contenter de ce qu'il est facile de dénoncer : si beaucoup de ces ouvrages critiquent l'essor du contrôle de gestion, peu entreprennent de «s'acquitter d'une étude systématique du travail de ces gestionnaires » (p. 106).

C'est, du reste, peut-être pour ne pas risquer de se voir reprocher de pratiquer la modération jusqu'à manquer de lucidité sur les dérives de la politique du chiffre, que sa conclusion invoque « une sollicitation de l'établissement bien supérieure aux moyens dont [il dispose] » (p. 255) et souligne que, « [c]oncrètement, il y a trop de chômeurs pour le peu de conseillers disponibles » (ibid.). On ne peut que suivre l'auteur quand il affirme que les personnels subissent un rationnement, aussi bien des offres d'emploi que du temps de travail, les conduisant à des « arbitrages de type gestionnaire entre des actes qu'ils jugent utiles et d'autres moins » (p. 256). Les personnels font au mieux pour faire un travail utile et qui conserve autant que possible du sens à leurs yeux. Mais un point notable est que la fusion autant que les logiques d'évaluation de la performance ne semblent pas avoir profondément affecté les identités professionnelles : « Le volet directement coercitif de la politique d'activation des dépenses de chômage n'est que peu investi par les conseillers à l'emploi », écrit l'auteur (p. 96). L'un des résultats qui mériteraient le plus de réflexion n'est-il pas celui-ci ? Alors que l'opérateur public de placement « a été reconfiguré de l'intérieur pour privilégier une approche gestionnaire de son activité par des acteurs qui faisaient profession de la mesure et de l'efficacité, les contrôleurs de gestion » (p. 27), les identités professionnelles héritées de l'ANPE n'ont pas réellement été transformées. Le travail est, pour ces agents, devenu plus coûteux à accomplir ; mais les logiques de performance ne l'ont pas modifié dans sa nature, au grand regret sans doute des plus libéraux et en relativisant les craintes des adversaires de ces derniers.

Au-delà, c'est dans l'articulation entre un travail ethnographique approfondi et une perspective historique qui n'est pas que de façade, que l'ouvrage trouve toute sa valeur. 
Cette perspective est des plus cruciales pour l'argument de l'ouvrage, notamment le rappel que c'est largement par son mouvement propre, afin de s'armer pour négocier avec sa tutelle, que l'ANPE s'est inscrite de façon précoce dans ce qui allait s'interpréter ultérieurement en termes de New Public Management. 